\title{
A Pilot Study of a Culturally-Appropriate, Educational Intervention to Increase Participation in Cancer Clinical Trials Among African Americans and Latinos
}

Jennifer Cunningham-Erves ( $\nabla$ jerves@mmc.edu )

Meharry Medical College https://orcid.org/0000-0002-7780-9874

Tilicia Mayo-Gamble

Georgia Southern University

Pamela C Hull

Vanderbilt University Medical Center

Tao Lu

Meharry Medical College

Claudia Barajas

Vanderbilt University Medical Center

Caree R. McAfee

Vanderbilt University Medical Center

Maureen Sanderson

Meharry Medical College

Juan R. Canedo

Meharry Medical College

Katina Beard

Matthew Walker Community Health Center

Consuelo H. Wilkins

Vanderbilt University Medical Center

\section{Research Article}

Keywords: cancer disparities, clinical trials, African Americans, Latinos, education, pilot project

Posted Date: April 29th, 2021

DOI: https://doi.org/10.21203/rs.3.rs-461056/v1

License: (c) (i) This work is licensed under a Creative Commons Attribution 4.0 International License. Read Full License 


\section{Abstract}

Aim: Culturally-appropriate, educational programs are recommended to improve cancer clinical trial participation among African Americans and Latinos. This study investigated the effect of a culturally-appropriate, educational program on knowledge, trust in medical researchers, and intent for clinical trial participation among African Americans and Latinos in Middle Tennessee.

Method: Trained community health educators delivered a 30-minute presentation with video testimonials to 198 participants in 13 town halls. A pre-post survey design was used to evaluate the intervention among 102 participants who completed both pre- and post-surveys one to two weeks after the session.

Results: Paired-sample t-test showed significant increases in unadjusted mean scores for knowledge $(p<.001)$, trust in medical researchers $(p<.001)$, and willingness to participate in clinical trials $(p=.003)$ after the town halls in the overall sample. After adjusting for gender and education, all three outcomes remained significant for the overall sample (knowledge: $p<.001$; trust in medical researchers: $p<.001$; willingness: $p=.001$ ) and for African Americans (knowledge: $p<.001$; trust in medical researchers: $p=.007$; willingness: $p=.005)$. However, willingness to participate was no longer significant for Latinos (knowledge: $p<.001$; trust in medical researchers: $p=.034$; willingness: $p=.084$ ).

Conclusions: The culturally-appropriate, educational program showed promising results for short-term, clinical trial outcomes. Further studies should examine efficacy to improve research participation outcomes.

\section{Introduction}

African American and Latino communities continue to suffer disproportionately from cancer health disparities. African Americans have the highest death rates and lowest survival rates for many cancers [1]. Trends indicate a growing cancer burden and existing disparities for certain cancer sites among Latinos [2]. Clinical trials and biospecimen-based research play a critical role in the efforts to reduce cancer rates and disparities. Clinical trials discover new detection methods, diagnostic tools, and life-sustaining treatments [3-4]. Biospecimen donations are the foundation for molecular and genetic studies of cancer prevention, diagnosis, and treatment [5-6]. Donations can be provided as a component of a clinical trial or as a donation to a biobank. However, less than $2 \%$ of minorities participate in clinical trials [7] and about $10 \%$ have donated biospecimens [8]. This warrants great concern as the favorable effects of new early detection methods and treatments may not be generalizable across all groups if racial and ethnic minority groups are not adequately represented in the studies [9-10]. This fuels existing cancer disparities through promulgating unequal benefits of scientific advances i.e., potentially promoting advances that favor socially advantaged groups and hindering advances that could improve outcomes for groups with the greatest needs [9-10]. Therefore, identifying barriers in recruitment is essential and a priority.

Many barriers exist on multiple levels that influence the recruitment of underrepresented minorities into clinical trials, including healthcare system-, physician-, and patient-level factors [10]. On the healthcare system level, examples of barriers are financial costs to the hospitals and their lack of resources to support clinical trials, limiting access to these trials. On the physician level, many lack knowledge on cancer clinical trial opportunities and time to present clinical trial information, decreasing chances of minorities being exposed to clinical opportunities [11]. Physicians may also have negative attitudes towards these trials or negative perceptions towards minority views or ability to participate in trials, which influences patient-provider communication [10]. On the patient level, some barriers the patient cannot control (i.e., lack of insurance, not meeting eligibility criteria) [10]. However, there are some barriers that can be addressed immediately. Low awareness and knowledge about research and opportunities for participation are the most commonly cited barriers among African Americans and Latinos [12]. Additional barriers are patient attitudes (e.g., mistrust in medical researchers, fear) and lack of culturally-targeted education [12-15]. Particularly, minorities have cited lower levels of knowledge and trust in medical researchers with higher levels of distrust among African Americans compared to Latinos [12, 15-16]. 
Few educational interventions on cancer clinical trials have been specifically designed to target racial/ethnic minorities, including African Americans and Latinos [17-28]. Only a handful can be used across both populations [17, 26]. Majority of these interventions are primarily for cancer patients in clinical settings to promote informed decision-making [18-21, 24$25,29]$. Results of these interventions have been inconsistent. Community-based interventions have been developed with similar purpose in creating change at the individual level with greater reach to a larger audience. The interconnected relationships within the community and their influence on decision-making allows participants of these interventions to promote familiarization with clinical trials through community advocacy [30]. These interventions have multi-components (e.g., computer-based-slide presentations, videos, brochures) [17, 22-23, 26-28, 31-33], and most have trained community health educators (CHEs) recruit and deliver these interventions. However, to the best of our knowledge, no culturallyappropriate interventions have assessed the impact of an intervention on trust in medical researchers and the research process.

Past research studies identified targeted, health communication as a strategy to promote participation in cancer clinical trials among African Americans and Latinos [6, 34-36]. Targeting can be used to develop a culturally-appropriate, educational program, and involves maximizing "fit" of information to a subgroup's unique characteristics [37]. Furthermore, researchers increasingly collaborate with CHEs to deliver health educational programs to the community [38]. Because they are well-trusted and respected within the community [39], CHEs can be leveraged to increase awareness on both cancer clinical trials participation and biospecimen donation.

\section{Educational Program}

Developed between 2014 to 2017, the educational program, "Clinical Trials: What's In It For Us?" is a 30-minute educational program. A multi-layered community-engagement process was used to develop the program while intricately interweaving community engagement to ensure the needs of the community were met. See Cunningham et al. for a full description of program development [40]. It consists of two culturally-appropriate slide presentations designed for African Americans and Latinos and video testimonials on cancer clinical trials followed by questions and answers. The video testimonials on cancer clinical trials were from community members and researchers who were African American and Latino. An additional Spanish version was available for the presentation and testimonial videos for the Latino community. Content covered in the slide presentation includes cancer etiology, risk factors, statistics, clinical trials, biospecimen donation, protections in research, and places to sign up for clinical trials. The program is limited to 20 participants to give the participants the opportunity to share their ideas and gain feedback on their questions.

In this pilot study, we examined the impact of a culturally-appropriate, educational program on short-term, clinical trial outcomes among African Americans and Latinos. The development of this educational program has been described previously [40]. We hypothesized that the program would lead to an increase in knowledge, trust in medical researchers, and willingness to participate in clinical trials within the overall sample and by race/ethnicity. Study findings will contribute to understanding of how culturally-appropriate programs can be used to improve short-term outcomes and inform future studies focused on clinical trials participation outcomes.

\section{Methods}

For this study, Meharry Vanderbilt Alliance (MVA) and Meharry-Vanderbilt-TSU Cancer Partnership (MVTCP) entered into a partnership with two community organizations, Matthew Walker Comprehensive Health Center (MWCHC) and Progreso Community Center (PCC) to conduct this community-engaged pilot study. MWCHC is the lead organization of the Nashville Health Disparities Coalition, serving primarily African Americans. PCC is the lead organization of the Nashville Latino Health Coalition, primarily serving Latinos. Collectively we worked to identify the best study design, recruitment and implementation strategies, and analyses for this work. 


\section{Study Design and Setting}

We conducted thirteen town halls in Nashville, Tennessee and surrounding counties to examine the effectiveness of the educational program entitled, "Clinical Trials: What's In It For Us?" A town hall is a group session that is conversational in nature and aimed to gain the community's input on health research priorities [41]. These townhalls took place in a variety of community-based organizations (i.e., churches, community centers, non-profit agencies, community health clinics). Community health educators led these townhalls. We used a pre-post quasi-experimental design to assess changes in short-term outcomes, which were knowledge about clinical trials, trust in medical researchers, and willingness to participate in clinical trials. This study was approved by Vanderbilt University Institutional Review Boards.

\section{Sampling and Recruitment}

A convenience sampling method was used to recruit participants. Partnership representatives and CHEs identified community members directly or they identified community-based organizations (CBOs) to recruit participants for each town hall. We intentionally stratified the sessions to identify organizations that would recruit primarily African American and Latino participants. Thirteen town halls represented African Americans $(n=7)$ and Latinos $(n=6)$. Recruitment methods were flyers and word-of-mouth. $\mathrm{CBO}$ s also made announcements on the study to the individuals they serve through email listservs, newsletters, and in-person meetings. The goal was to recruit on average 20 participants per town hall.

\section{Eligibility Criteria}

For this study, inclusion criteria were: 1) female or male; 2) aged 18 and older; 3) self-identified African American or Latino; and 4) English-speaking if African American and Spanish/English speaking if Latino.

\section{Program Description}

The clinical trial educational program "Clinical Trials: What's In It For Us?" is a 25-slide presentation designed to be culturally appropriate for African Americans and Latinos. The program consists of 2 presentations which include video testimonials from African American and Latino community members and researchers. A Spanish version was also available for the Latino community. The content of the presentation included basic cancer information including a definition of cancer, risk factors for cancer, statistics on how cancer affects the community, top three cancers by gender and race, and why clinical trials are important in cancer prevention and control. Content related to cancer clinical trials involved definition of clinical trials and their importance. Additional content included successes, risks, benefits, process, and patient protections in research. To ensure the content was culturally-appropriate (e.g., use of dominant language or images reflective of the target audience), we had a diverse team (1 MD, $4 \mathrm{PhD}$ ) with extensive knowledge and experience in community engagement, communication, and/or cancer. Two community leaders and two community health educators were also engaged throughout the process to ensure cultural sensitivity. Post-development, scientific experts and community members reviewed the presentation to ensure relevance, accuracy, and comprehensiveness. See Cunningham et al. [41] for a full description of the development process and Figures 1 and 2 for sample content.

\section{Training Community Health Educators (CHEs)}

To implement the program in the pilot study, we trained or re-trained six CHEs [40] in a half day training. CHEs were selected based on the following criteria: (1) located throughout Middle Tennessee; (2) embedded community members with trusted relationships throughout the community; and (3) key players in fostering healthy behaviors in their communities. Training involved an overview of cancer, types of cancer, and prevention including clinical trials, as well as learning presentation styles and use of the evaluation survey. The training was facilitated by members of the research team with extensive years of experience in implementing health education programs for minority populations. The 
training evaluation included trainee practice sessions. The trainee had to demonstrate mastery in the following four areas: 1) understanding of cancer disease and how to prevent it; 2) defining clinical trials and their process; 3 ) describing the benefits and risks of clinical trial participation; and 4) discussing the process of clinical trial enrollment and participant protections. CHEs were provided feedback to improve presentation delivery, as needed. In addition to the half-day training specific to this program,the CHEs attended additional trainings and workshops as part of their overall $\mathrm{CHE}$ training and job responsibilities to learn more about different types of cancer, preventive behaviors, and strategies to engage the community. They also regularly developed and implemented cancer education programs in the community on different cancer types and preventive behaviors. Collectively, these trainings also prepared the CHEs to be able to lead these town halls meetings.

\section{Survey Measures}

We developed a survey to measure clinical trial knowledge, trust in medical research, and willingness to participate in clinical trials that was administered pre- and post-town halls. These measures are described below.

Knowledge. The clinical trial knowledge scale, a validated scale by Ma et al., has 22-items with questions on knowledge and beliefs related to clinical trials [42]. These statements were presented with response options as yes, no, or not sure. For this study, we used the thirteen questions which directly related to knowledge. Example statements included, "I understand what clinical trials are." and "I know that a percentage of patients may get a placebo." A sum of correct answers was produced with scale score ranges from 0 to 13. Cronbach's alpha in this sample was 0.78 .

Trust. The 12-item trust in medical researchand researchers scale, a validated scale, measures the level of trust the public has towards biomedical research in general [43]. Questions were assessed using a 5-point Likert scale based on agreement. A final score was produced by summing all individual item scores, with final scores ranging from 12 to 60 and higher scores indicating a greater degree of trust towards medical research. Cronbach's alpha in this sample was 0.72 . Example statements include, "Doctors who do medical research are only about what is best for each patient." and "Medical researchers treat people like guinea pigs".

Willingness. The willingness to participate in clinical trials was assessed using a single item created by the research team, "What is the likelihood of you taking part in a clinical trial in the future?". The response options were yes, no, and not sure. Answer choices "no" and "not sure" were collapsed into one category for analysis.

Socio-demographics. Participants self-identified their race/ethnicity as African American or Latino. Gender was represented with the categories of male and female. Education levels included no high school diploma, GED or high school diploma, some college, associates' degree, bachelors' degree, masters' degree, and doctoral degree. For analysis, education was collapsed into two categories: some college or lower, versus bachelor's degree of higher.

\section{Procedures}

The educational program was led by a trained $\mathrm{CHE}$, who was matched to the targeted group for each town hall (i.e., African American or Latino). The same CHE facilitated the town halls among each group. The town halls lasted approximately 90 minutes, dependent on the amount of discussion. Prior to the presentation, the $\mathrm{CHE}$ provided an explanation of the program and provided a copy of the slide presentation obtained informed consent, and asked participants to complete the pre-survey on paper. After the presentation and videos, there was a question and answer session. Participants were then provided culturally-appropriate printed educational brochures on cancer and preventive behaviors. When available, Announcements or flyers were provided on other educational programs or research opportunities. Last, CHEs provided contact information to serve as an educational resource and ultimately build and sustain trust. One to two weeks post-town halls, the CHE's conducted the post-survey by phone based on participants' availability. 


\section{Analysis}

For descriptive purposes, demographic characteristics were summarized by race/ethnicity for the overall sample, among participants who completed the pre-survey only, and among participants who completed both the pre- and post-surveys. Data analyses were conducted on those who completed the pre- and post-tests. Paired samples t-tests for continuous variables and McNemar's test for categorical/binary variables determined univariate differences between pre- and postsurveys of trust, knowledge and willingness for: 1) the whole sample; 2) African Americans only; and 3) Latinos only. Multivariate regression models were used to estimate the associations between pre- and post-assessments of each outcome variable while adjusting for sociodemographic variables (i.e., race, gender, education). Linear regression was used for knowledge and trust as continuous variables, and logistic regression was used for willingness as a binary variable. The models were estimated for the total sample then separately for African Americans and Latinos, excluding the race/ethnicity variable as a covariate. A significance level of alpha $=.05$ was selected. A portion of analysis were done with SPSS version 25 and SAS 9.4 was used for additional analyses in more detail.

\section{Results}

A total of 198 participants completed the program; 116 were African American and 82 were Latino. Of these, 104 participants completed the pre-test and post-test surveys, yielding $51.5 \%$ overall response rate. When comparing those who completed the pre-test only versus those who completed both pre- and post-test surveys, there was a difference by race $(p=.001)$, with African Americans being less likely to complete the post-test than Latinos (42.2\% versus $67.1 \%$ ). However, there were no significant differences by education, gender, marital status, and employment (Results not shown).

Table 1 provides demographic characteristics of the study sample that completed pre- and post-test surveys. Among African Americans, the majority were female (59.6\%), married (50.0\%), employed full-time or retired (70.2\%), and had a college degree or higher (55.1\%). Among Latinos, the majority were female (85.7\%), married (81.4\%), were employed fulltime or part-time (50.0\%), and had some college or less (68.0\%). There were significant differences in gender $(\chi=7.361 ; p$ $=.009)$, marital status $(X=26.099 ; p<.001)$, employment status $(\chi=50.781 ; p<.001)$, and education $(\chi=11.795 ; p=.001)$ by race.

\section{Univariate Pre-Post Analysis}

Table 2 summarizes the outcome variables (i.e., knowledge on clinical trials, trust in medical researchers, and willingness to participate in medical research) and tests for pre- and post- intervention changes. In the overall sample, significant increases were observed for knowledge on cancer clinical trials $(t=-8.38 ; p<.001)$, trust in medical researchers $(t=-3.88$; $p<.001)$, and willingness to participate in clinical trials $\left(X^{2}=8.76 ; p=.003\right)$ one to two weeks after the town halls. In the subsample of African Americans, all three outcomes also increased significantly at post-intervention follow-up knowledge on cancer clinical trials $(t=-4.89 ; p<.001)$, trust in medical researchers $(t=-4.19 ; p<.001)$, and their willingness to participate in cancer clinical trials $\left(X^{2}=5.40 ; p=.02\right)$. Latinos demonstrated significant increase in knowledge on cancer clinical trials $(t=-6.98 ; p<.001)$, but the increases in trust in medical researchers $(t=-1.48 ; p=.145)$ and willingness to participate in cancer clinical trials were not statistically significant $\left(X^{2}=3.55 ; p=.059\right)$ (See Table 2$)$.

\section{Multivariate Regression Analysis}

In the multivariate analysis, we assessed whether the pre-post differences in knowledge, trust, and willingness to participate as they relate to clinical trials would remain after we adjusted for gender, race/ethnicity, and education (Table $3)$. In the overall sample, knowledge scores on average increased by 1.91 for all participants $(p<.001)$. As it relates to education, knowledge scores of participants with a college degree or higher increased 1.63 on average compared to individuals with less than a college degree $(p<.001)$. For race, Latinos level of knowledge on average was 1.21 lower than 
African Americans. Scores for trust in medical researchers on average increased by 2.26 points for all participants $(p<$ .001), in which trust levels of Latinos on average was 1.65 higher than African Americans. Last, participants were 2.45 times more likely to indicate willingness to participate in clinical trials post-intervention than pre-intervention $(p=.001)$. Participants with college level or more education were 2.31 times more likely to indicate willingness to participate in clinical trials post-intervention than pre-intervention $(p=.007)$. For race, Latinos were 2.38 times more likely to indicate willingness to participate in clinical trials compared to African Americans post intervention compared to pre-intervention $(p=.003)$.

Among African Americans, the significant differences in pre and post knowledge, trust, and willingness remained when controlling for gender and education. For all participants, knowledge levels increased 1.77 on average $(p<.001)$. As it relates to education, participants with a college degree or higher increased 1.73 on average compared to individuals with less than a college degree $(p<.001)$. Trust levels on average increased 2.79 points for all participants $(p=.007)$. No demographic variables were associated with trust. Participants who stated they were unwilling to participate in a clinical trial pre-intervention were 2.99 times more likely to be willing to participate post-intervention $(p=.005)$. In comparing willingness scores pre-post intervention, females were 0.39 times more likely to be willing to participate compared to men $(p=.012)$. Last, those with college or more education were 2.34 times more likely to be willing to participate postintervention compared to pre-intervention $(p=.018)$.

Among Latinos, knowledge $(p<.001)$ remained significant after controlling for sociodemographic variables. However, trust $(p=.034)$ became significant after controlling for sociodemographic variables. There was no significant increase in willingness to participate in clinical trials among Latinos, even after adjusting for gender and education $(p=0.84)$. Gender and education were not significantly associated with any of the outcome variables for Latinos.

\section{Discussion}

We assessed the impact of a culturally-appropriate, educational intervention on knowledge, trust, and willingness to participate in clinical trials among African Americans and Latinos. No other studies have reported evaluation of an educational program that targets both groups. Furthermore, no other studies have assessed the effect of cancer clinical trial educational programs, such as ours, on trust in medical researchers and the research process among African Americans and Latinos. Hence, this study makes an important contribution to the literature.

Culturally-targeted programs are important as there is a long-standing history of mistrust in researchers and the research process among these racial minority groups [44], which contributes to low participation rates in clinical trials [45]. In our overall sample, we observed a significant increase in levels of trust post intervention. However, when studying the effect of the program on trust by race, there was only a significant increase in trust for African Americans. Past research suggests and demonstrates use of community engaged research (CEnR) principles as an effective strategy for increasing trust among underrepresented research groups $[46,47]$. While our results confirm these findings among the African American study participants, we were surprised to find that trust levels were not influenced among the Latino study participants. It is important to note that the mean trust score indicated Latinos had higher levels of trust compared with African Americans pre- and post-intervention. Because there was a significant difference at baseline in trust between African American and Latinos, there may have been a ceiling effect of having limited room for increase among Latinos whereas African Americans started with lower scores, allowing more room to increase. Regardless, these results suggest that either there are additional factors influencing trust or additional strategies should be considered when addressing the Latino population on clinical trials. While we adjusted for gender and education, future research could explore a potential moderating effect for these variables on intervention outcomes, since neither had significant direct effects in adjusted models. 
Results demonstrated that participants increased their levels of knowledge and were more willing to participate in cancer clinical trials post-intervention. These results confirmed our hypothesis and are in line with past research that has demonstrated cancer clinical trial educational programs increased awareness, knowledge, and attitudes towards research $[17,22-23,32]$. For example, Green et al. found that a culturally-tailored, educational module using three formats (i.e., Call and Response, Role-Play, and Workshop) significantly increased awareness on cancer clinical trials, access to clinical trial opportunities, and improved intentions to engage in decision-making regarding cancer clinical trials [23]. Therefore, these results confirm that cancer clinical trial educational programs on the community level are important for addressing one of the most prominent barriers to cancer clinical trial participation - low knowledge and awareness of clinical trials and opportunities.

The sociodemographic factors assessed in this study were race, gender, and education. For trust, we did not find any sociodemographic variables associated with post-intervention trust levels in the overall sample or by race. This suggests that the increase in levels of trust could be a direct result of our intervention, although the potential intervention effect needs to be confirmed in a randomized controlled trial. Education remained significant as a covariate for knowledge only among African Americans, not Latinos. It is not surprising that higher education is associated with higher knowledge, but it is interesting that it did not have a significant association for Latinos. Possibly, those with higher educational levels were able to understand the intervention at greater levels compared to those with lower education attainment, reflecting the change in knowledge. A second alternative is the information may have been presented at levels in which those with lower education levels could understand. Therefore, future research should be conducted to gain insight on the moderating effect of education.

Last, unsurprisingly, post-intervention willingness was associated with education and race in the overall sample with participants. Specifically, those with higher education and were Latino had greater willingness post-intervention compared to those with lower education and were African American. In the subgroup analyses, education remained significant and gender became significant among African Americans. Specifically, those who were female and higher education had greater willingness compared to men and those with lower education. However, education was not significantly associated with willingness among Latinos. Therefore, educational attainment alone may not explain variation in the willingness of Latinos to participate in clinical trials. Future studies should explore if there are additional factors influencing willingness to participate in clinical trials that should be controlled for as covariates.

This study has several strengths. First, we demonstrate the success in applying CEnR principles to implement a culturallyappropriate intervention to improve knowledge, attitudes, and willingness to participate in clinical trials among a high-risk group. Second, we determined that a culturally-appropriate, educational intervention had a significant impact on trust among African Americans, which is an underrepresented research group with a history of distrust in medical researchers and the research process [44]. However, this study is not without limitations. First, participants were recruited using a convenience sample. Therefore, findings may not be generalizable to a specific population and selection bias could have been introduced in the study. Next, there is a possible risk of bias due to social desirability in self-reported responses. Additionally, the study design did not include a control group. Instead, the pre-post design allowed participants to serve as their own controls. Furthermore, we did not assess cancer knowledge or experiences with cancer, the healthcare system, or researchers, which are also factors that could have an influence on outcomes. We will explore these components in future studies with larger samples that will enable the inclusion of more variables in multivariate models. Finally, our study had a low follow-up response rate and a short timeframe for follow-up, ranging from one week to two weeks. The low response rate could be attributed to the timing of post-survey data two weeks post-intervention. Furthermore, we did not incentivize participants to take the post-survey. To overcome some of these limitations, future studies should test this intervention using a randomized controlled design, conduct immediate post-session assessments and longer-term follow-up assessments to examine maintenance of intervention effects, and offer incentives or different modes of delivery for follow-up survey to potentially boost response rates. 
Despite these limitations, this is one of the first pilot studies exclusively focused on increasing clinical trial enrollment among African Americans and Latinos. These results demonstrate promise in the use of this culturally-appropriate educational program to improve cancer clinical trial participation among these groups. Findings from this study will serve as preliminary data for a larger, well-powered randomized controlled trial to assess efficacy in the short-term outcomes examined here, as well as longer-term behaviors related to actual research participation.

\section{Declarations}

\section{Funding}

This work was supported in part by Meharry-Vanderbilt Alliance, Vanderbilt-Ingram Cancer Center and National Institutes of Health (NIH) grant numbers P30 CA068485, P30 CA068485-23S5, U54 CA163072, U54 CA163069, U54 CA163066, UL1 RR024975 and UL1 TR000445.

\section{Conflicts of interest/Competing interests}

The authors declare that they have no conflict of interest.

\section{Availability of data and material}

The data are not publicly available due to the inclusion of information that could compromise research participant privacy/consent.

\section{Authors' contributions}

Consuelo H. Wilkins, Maureen Sanderson, Pamela C. Hull, Katina Beard, and Jennifer Cunningham-Erves participated in the conception and design of the study. Consuelo $\mathrm{H}$. Wilkins was responsible for obtaining ethics approval and acquiring funding, and Jennifer Cunningham-Erves maintained ethics approval. Jennifer Cunningham-Erves and Consuelo $\mathrm{H}$. Wilkins managed the study. All authors contributed to pilot study development. Jennifer Cunningham-Erves and Claudia Barajas recruited and screened eligible patients and collected data. Jennifer Cunningham-Erves, Tilicia Mayo-Gamble, and Claudia Barajas were managed the data. Jennifer Cunningham-Erves, Tilicia Mayo-Gamble, and Tao Lu conducted data analysis, and Tao Lu, Jennifer Cunningham-Erves, Tilicia Mayo-Gamble, Pamela C. Hull and Maureen Sanderson interpreted results. Jennifer Cunningham-Erves drafted the manuscript. All authors revised the manuscript and have read and approved the final manuscript.

\section{Ethics Approval}

All study procedures involving human participants were in accordance with the ethical standards of the institutional and/or national research committee and with the 1964 Helsinki Declaration and its later amendments or comparable ethical standards. The study was approved by the Bioethics Committee of Vanderbilt University (IRB \#: 171110).

\section{Consent to participate}

Informed consent was obtained from all individual participants included in the study.

Acknowledgements: We would like to thank the partner community-based organizations in the greater Nashville, Tennessee, area who collaborated with us to identify interested community members to participate in this study.

\section{References}


1. DeSantis CE, Miller KD, Goding Sauer A, Jemal A, Siegel RL (2019) Cancer statistics for African Americans, 2019. CA Cancer J Clin69(3):211-233. doi 10.3322/caac.21555

2. Miller KD, Goding Sauer A, Ortiz AP, et al (2018) Cancer Statistics for Hispanics/Latinos, 2018. CA Cancer J Clin 68(6):425-445. doi: 10.3322/caac.21494

3. Murthy VH, Krumholz HM, Gross CP (2004) Participation in cancer clinical trials: race-, sex-, and age-based disparities. JAMA 291(22):2720-2726. doi: 10.1001/jama.291.22.2720

4. Tejeda HA, Green SB, Trimble EL, et al (1996) Representation of African-Americans, Hispanics, and whites in National Cancer Institute cancer treatment trials. J Natl Cancer Inst 88(12):812-816.doi: 10.1038/s41408-018-0102-7

5. Ambrosone CB, Nesline MK, Davis W (2006) Establishing a cancer center data bank and biorepository for multidisciplinary research. Cancer Epidemiol Biomarkers Prev.15(9):1575-1577.doi: 10.1158/1055-9965.EPI-06-0628

6. Dang JH, Rodriguez EM, Luque JS, Erwin DO, Meade CD, Chen MS, Jr (2014) Engaging diverse populations about biospecimen donation for cancer research. J Community Genet 5(4):313-327.doi: 10.1007/s12687-014-0186-0

7. Chen MS, Jr., Lara PN, Dang JH, Paterniti DA, Kelly K (2014) Twenty years post-NIH Revitalization Act: enhancing minority participation in clinical trials (EMPaCT): laying the groundwork for improving minority clinical trial accrual: renewing the case for enhancing minority participation in cancer clinical trials. Cancer120 Suppl 7:1091-1096. doi: $10.1002 /$ cncr.28575

8. Simon MA, de la Riva EE, Bergan R, et al (2014) Improving diversity in cancer research trials: the story of the Cancer Disparities Research Network. J Cancer Educ29(2):366-374. doi: 10.1007/s13187-014-0617-y

9. George S, Duran N, Norris K (2014) A systematic review of barriers and facilitators to minority research participation among African Americans, Latinos, Asian Americans, and Pacific Islanders. AmericanJournal of Public Health 104(2):e16e31.doi: 10.2105/AJPH.2013.301706

10. Hamel LM, Penner LA, Albrecht TL, Heath E, Gwede CK, Eggly S (2016) Barriers to Clinical Trial Enrollment in Racial and Ethnic Minority Patients With Cancer. Cancer Control 23(4):327-337.doi: 10.1177/107327481602300404

11. Hillyer GC, Beauchemin M, Hershman DL, et al (2020) Discordant attitudes and beliefs about cancer clinical trial participation between physicians, research staff, and cancer patients. Clin Trials 17(2):184-194. doi:

$10.1177 / 1740774520901514$

12. Rivers DA, Pal T, Vadaparampil ST, Adams LA, Dash-Pitts L, Quinn GP (2019) A community-academic partnership to explore informational needs of African American women as a primer for cancer clinical trial recruitment. Ethn Health24(6):679-693. doi: 10.1080/13557858.2017.1367762

13. Unger JM, Cook E, Tai E, Bleyer A (2016) The Role of Clinical Trial Participation in Cancer Research: Barriers, Evidence, and Strategies. Am Soc Clin Oncol Educ Book 35:185-198.

doi: 10.1200/EDBK_156686

14. Friedman DB, Foster C, Bergeron CD, Tanner A, Kim SH (2015) A qualitative study of recruitment barriers, motivators, and community-based strategies for increasing clinical trials participation among rural and urban populations. Am $\mathrm{J}$ Health Promot 29(5):332-338.doi: 10.4278/ajhp.130514-QUAL-247 
15. Brandberg Y, Johansson H, Bergenmar M (2016) Patients' knowledge and perceived understanding - Associations with consenting to participate in cancer clinical trials. Contemp Clin Trials Commun2:6-11. doi: 10.1016/j.conctc.2015.12.001

16. Friedman DB, Bergeron CD, Foster C, Tanner A, Kim SH (2013) What do people really know and think about clinical trials? A comparison of rural and urban communities in the South. J Community Health 38(4):642-651. doi:

10.1007/s10900-013-9659-z

17. Pelto DJ, Sadler GR, Njoku O, et al (2016) Adaptation of a Cancer Clinical Trials Education Program for African American and Latina/o Community Members. Health Educ Behav 43(4):381-388. doi: 10.1177/1090198115610555

18. Chalela P, Muñoz E, Gallion KJ, Kaklamani V, Ramirez AG (2018) Empowering Latina breast cancer patients to make informed decisions about clinical trials: a pilot study. Transl Behav Med8(3):439-449. doi: 10.1093/tbm/ibx083

19. Du W, Mood D, Gadgeel S, Simon MS (2008) An educational video to increase clinical trials enrollment among lung cancer patients. J Thorac Oncol3(1):23-29. doi: 10.1097/JT0.0b013e31815e8bb2

20. Du W, Mood D, Gadgeel S, Simon MS (2009) An educational video to increase clinical trials enrollment among breast cancer patients. Breast Cancer Res Treat 117(2):339-347.doi: 10.1007/s10549-009-0311-7

21. Fouad MN, Acemgil A, Bae S, et al (2016) Patient Navigation As a Model to Increase Participation of African Americans in Cancer Clinical Trials. J Oncol Pract 12(6):556-563.doi: 10.1200/JOP.2015.008946

22. Frew PM, Schamel JT, O'Connell KA, Randall LA, Boggavarapu S (2015) Results of a Community Randomized Study of a Faith-Based Education Program to Improve Clinical Trial Participation among African Americans. Int J Environ Res Public Health 13(1):ijerph13010041.doi: 10.3390/ijerph13010041

23. Green MA, Michaels M, Blakeney N, et al (2015) Evaluating a community-partnered cancer clinical trials pilot intervention with African American communities. J Cancer Educ 30(1):158-166.doi: 10.1007/s13187-014-0764-1

24. Robinson BN, Newman AF, Tefera E, et al (2017) Video intervention increases participation of black breast cancer patients in therapeutic trials. NPJ Breast Cancer3:36. doi: 10.1038/s41523-017-0039-1

25. Skinner JS, Fair AM, Holman AS, Boyer AP, Wilkins CH (2019) The Impact of an Educational Video on Clinical Trial Enrollment and Knowledge in Ethnic Minorities: A Randomized Control Trial. Front Public Health 7:104. doi:

10.3389/fpubh.2019.00104

26. Sadler GR, Gonzalez J, Mumman M, et al (2010) Adapting a Program to Inform African American and Hispanic American Women about Cancer Clinical Trials. J Cancer Educ25(2):142-145.doi: 10.1007/s13187-009-0032-y

27. Blakeney N, Michaels M, Green M, et al (2015) Collaborative development of clinical trials education programs for African-American community-based organizations. J Cancer Educ30(2):400-406. doi: 10.1007/s13187-014-0673-3

28. Ford M, Wahlquist A, Blake R, et al (2014) Assessing an Intervention to Improve Clinical Trial Perceptions Among Predominately African-American Communities in South Carolina. Prog Community Health Partnersh6(3):249-263. doi: 10.1353/cpr.2012.0038

29. Lucas T, Hayman LW, Jr., Blessman JE, Asabigi K, Novak JM. Gain versus loss-framed messaging and colorectal cancer screening among African Americans: A preliminary examination of perceived racism and culturally targeted dual messaging. Br J Health Psychol. 2016;21(2):249-267. doi: 10.1111/bjhp.12160 
30. McLeroy KR, Norton BL, Kegler MC, Burdine JN, Sumaya CV. Community-based interventions. Am J Public Health. 2003;93(4):529-533. doi: 10.2105/ajph.93.4.529

31. Kiviniemi MT, Saad-Harfouche FG, Ciupak GL, et al (2013) Pilot intervention outcomes of an educational program for biospecimen research participation. J Cancer Educ28(1):52-59.

doi: 10.1007/s13187-012-0434-0

32. Patel K, Inman W, Gishe J, et al (2018) A Community-Driven Intervention for Improving Biospecimen Donation in African American Communities. J Racial Ethn Health Disparities5(1):15-23. doi: 10.1007/s40615-017-0336-2

33. Rodriguez EM, Saad-Harfouche FG, Miller A, et al (2016) Engaging diverse populations in biospecimen donation: results from the Hoy y Manana study. J Community Genet7(4):271-277. doi: 10.1007/s12687-016-0275-3

34. Zuniga ML, Blanco E, Martinez P, Strathdee SA, Gifford AL (2007) Perceptions of barriers and facilitators to participation in clinical trials in HIV-positive Latinas: a pilot study. J Womens Health (Larchmt) 16(9):1322-1330. doi: 10.1089/jwh.2006.0234

35. Davis TC, Arnold CL, Mills G, Miele L (2019) A Qualitative Study Exploring Barriers and Facilitators of Enrolling Underrepresented Populations in Clinical Trials and Biobanking. Front Cell Dev Biol 7:74.doi: 10.3389/fcell.2019.00074

36. Heredia NI, Krasny S, Strong LL, et al (2017) Community Perceptions of Biobanking Participation: A Qualitative Study among Mexican-Americans in Three Texas Cities. Public Health Genomics 20(1):46-57.doi: 10.1159/000452093

37. Kreuter MW, Wray RJ (2003) Tailored and targeted health communication: strategies for enhancing information relevance. American journal of health behavior 27 Suppl 3:S227-232.

doi: 10.5993/ajhb.27.1.s3.6

38. Hohl SD, Thompson B, Krok-Schien JL, et al (2016) Characterizing Community Health Workers on Research Teams: Results from Centers for Population Health and Health Disparities. Am J Public Health 106(4):664-670. doi: 10.2105/AJPH.2015.302980

39. Malcarney MB, Pittman P, Quigley L, Horton K, Seiler N (2017) The Changing Roles of Community Health Workers. Health Ser Res 52:360-382. doi: 10.1111/1475-6773.12657

40. Cunningham-Erves J, Barajas C, Mayo-Gamble T, et al (2020) Formative Research to Design a Culturally-Appropriate Cancer Clinical Trial Education Program to Increase Participation of African American and Latino Communities. BMC Public Health 20:840. http://doi.org/10.1186/s12889-020-08939-4

41. Etchegary H, Bishop L, Street C, et al (2017) Engaging patients in health research: identifying research priorities through community town halls. BMC Health Serv Res17:192. doi: 10.1186/s12913-017-2138-y

42. Ma GX, Tan Y, Blakeney NC, et al (2014) The impact of a community-based clinical trial educational intervention among underrepresented Chinese Americans. Cancer Epidemiol Biomarkers Prev23(3):424-432. doi: 10.1158/10559965.EPI-13-0773

43. Hall MA, Camacho F, Lawlor JS, Depuy V, Sugarman J, Weinfurt K (2006) Measuring trust in medical researchers. Med Care44(11):1048-1053. doi: 10.1097/01.mlr.0000228023.37087.cb 
44. Scharff DP, Mathews KJ, Jackson P, Hoffsuemmer J, Martin E, Edwards D (2010) More than Tuskegee: understanding mistrust about research participation. J Health Care Poor Underserved 21(3):879-897.doi: 10.1353/hpu.0.0323

45. Durant RW, Legedza AT, Marcantonio ER, Freeman MB, Landon BE (2011) Willingness to participate in clinical trials among African Americans and Whites previously exposed to clinical research. J Cult Divers 18(1):8-19.

46. Sankaré IC, Bross R, Brown AF, et al (2015) Strategies to Build Trust and Recruit African American and Latino Community Residents for Health Research: A Cohort Study. Clin Transl Sci 8(5):412-420. doi: 10.1111/cts.12273

47. Clinical and Translational Science Awards Consortium Community Engagement Key Function Committee Task Force on the Principles of Community Engagement (2011) Principles of Community Engagement 2nd Edition. National Institute of Health, Bethesda, Maryland.

\section{Tables}

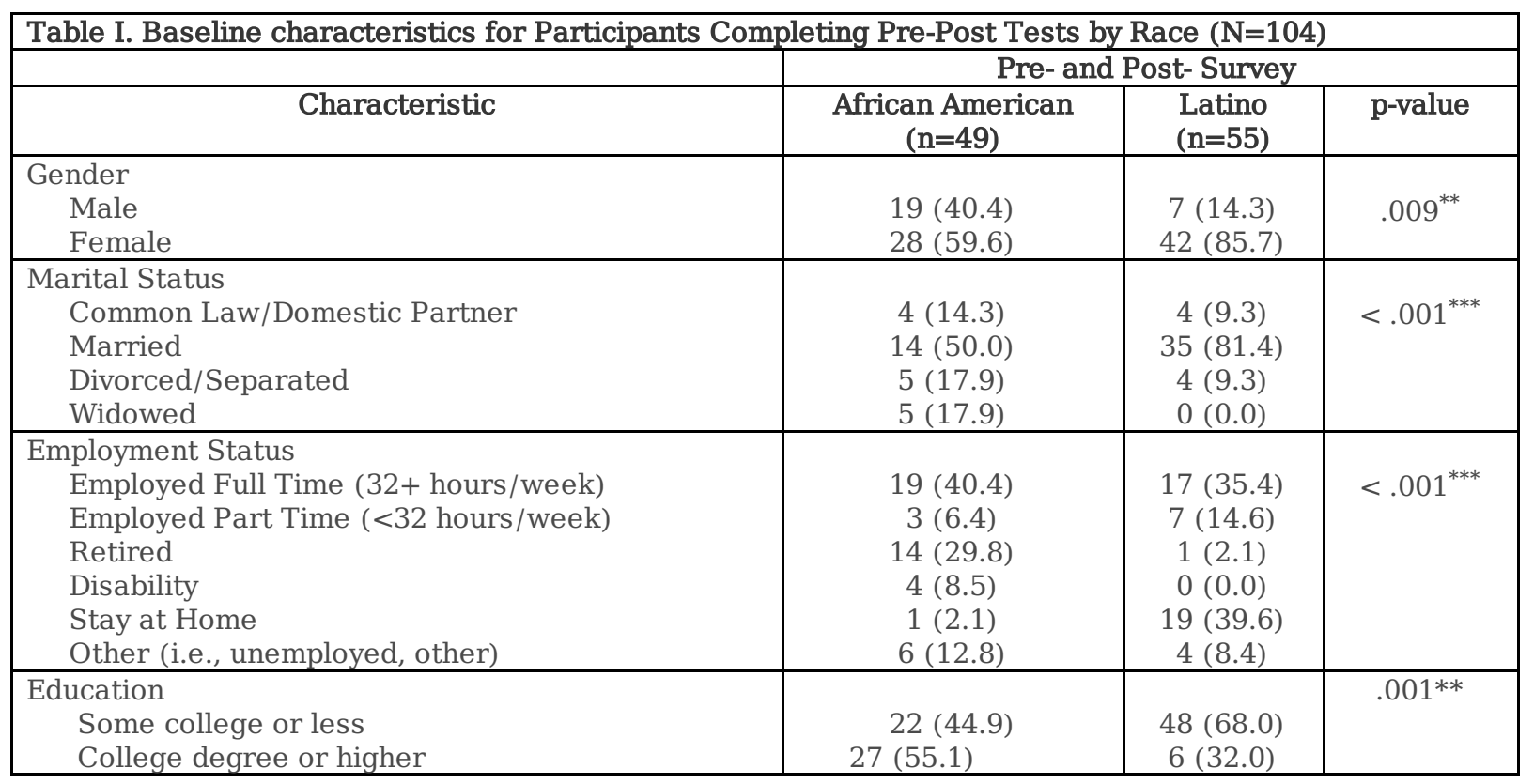

Note: $\quad{ }^{*} p<.05 ;{ }^{* *} p<.01 ;{ }^{* * *} p<.01$; Numbers per category may not equal total $\mathrm{n}$ due to missing values. 
Table II. Pre-post changes in knowledge, trust in medical researchers, and willingness to participate in clinical trials (unadjusted), overall and by race

\section{Paired Samples T-Test}

Pre Post t-test P-value

Mean SD Mean SD

\section{Overall}

\begin{tabular}{lcccccc} 
Trust in Medical Researchers & 39.48 & 5.97 & 41.57 & 5.06 & -3.88 & $<.001^{\star \star \star}$ \\
\hline Knowledge & 6.27 & 2.94 & 8.49 & 2.65 & -8.38 & $<.001^{\star \star \star}$
\end{tabular}

\section{African Americans}

Trust in Medical Researchers

37.25

6.09

40.43

5.54

$-4.19$

$<.001^{\star \star \star}$

Knowledge

7.53

2.94

9.49

2.71

$-4.89$

$<.001^{\star \star *}$

\section{Latinos}

Trust in Medical Researchers

41.51

5.12

42.61

4.38

$-1.48$

.145

Knowledge

5.15

2.46

7.60

2.28

$-6.98$

$<.001^{\star \star \star}$

McNemar Test: Percent of People Willing to Participate in Research, Pre Test and Post Test

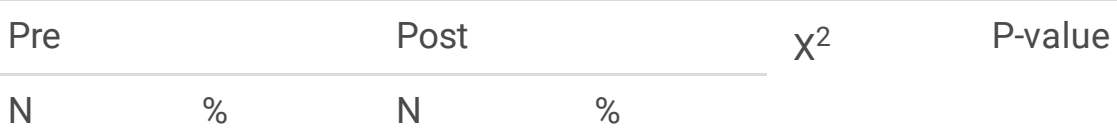

\section{Overall}

Willing to Participate

47

46.53

64

63.37

8.76

$.003^{* *}$

African Americans

Willing to Participate

20

42.55

29

61.70

5.40

$.020^{\star}$

Latinos ( $\mathrm{n}=54)$

Willing to Participate

27

50.00

35

64.81

3.555

.059

Note: $\quad{ }^{*} p<.05 ;{ }^{\star *} p<.01 ;{ }^{* \star *} p<.001$ 
Table III. Multivariate regression estimates of pre-post changes in clinical trial outcomes, adjusting for demographic variables, overall and by race

\section{Total Sample}

b S.E. tratio

\section{African American}

p-value b S.E. t-

\section{Latino}

p-value $b \quad$ S.E. $t^{-} \quad$ p-value ratio ratio

\section{Knowledge}

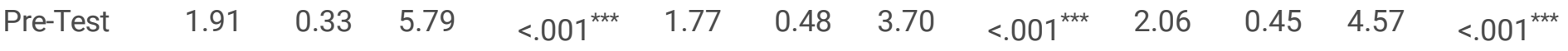

Gender

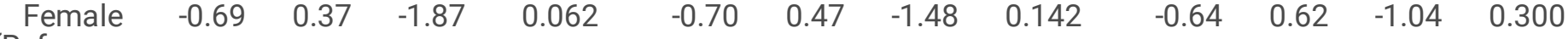
(Ref:

Male)

Education

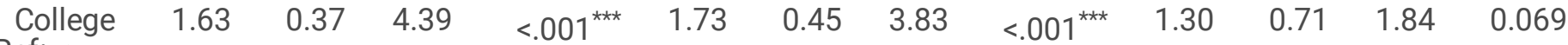
(Ref: $<$ College)

Race

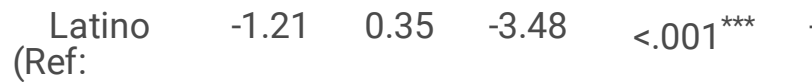

African

American)

\section{Trust in Medical Researchers}

Pre-Test

$\begin{array}{lll}2.26 & 0.68 & 3.34\end{array}$

$<.001^{\star \star \star} \quad 2.79$

$0.007^{\star \star} \quad 1.81$

$0.84 \quad 2.14$

$0.034^{\star *}$

Gender

Female

(Ref:

Male)

Education

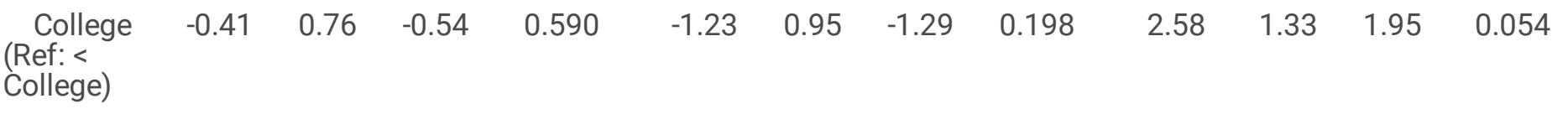

Race

$\begin{array}{lllll}\text { Latino } & 1.65 & 0.72 & 2.30 & 0.022^{*}\end{array}$

African

American)

Total Sample

OR S.E. $\quad X^{2} \quad$ p-value
African American

Latino

OR $\quad$ S.E. $\quad x^{2} \quad$ p-value $\quad$ OR $\quad$ S.E. $\quad x^{2} \quad$ p-value

Willingness to Participate in Clinical Trials

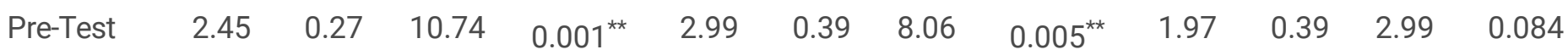

Gender

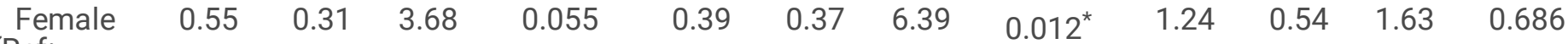

(Ref:

Male) 
Education

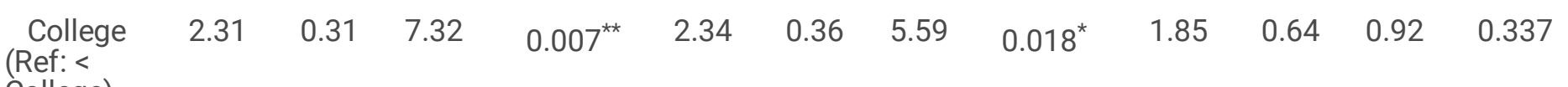
College)

Race

\begin{tabular}{|c|c|c|c|}
\hline $\begin{array}{l}\text { Latino } \\
\text { (Ref: } \\
\text { African } \\
\text { American) }\end{array}$ & 2.38 & 0.29 & 8.71 \\
\hline
\end{tabular}

Note: $\quad{ }^{*} p<.05 ;{ }^{* *} p<.01 ;{ }^{* * *} p<.001$

\section{Figures}

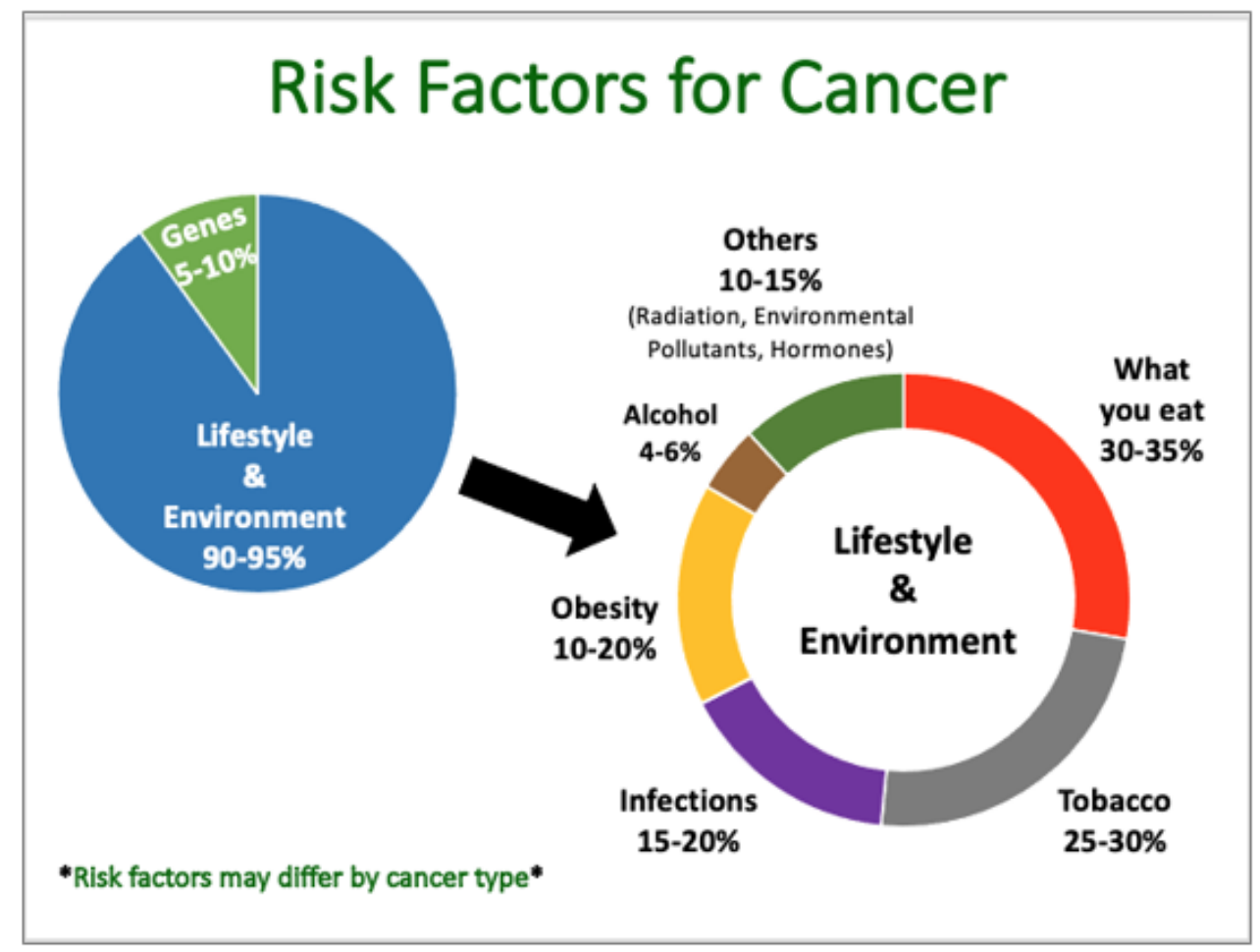

Figure 1

Sample content on cancer 


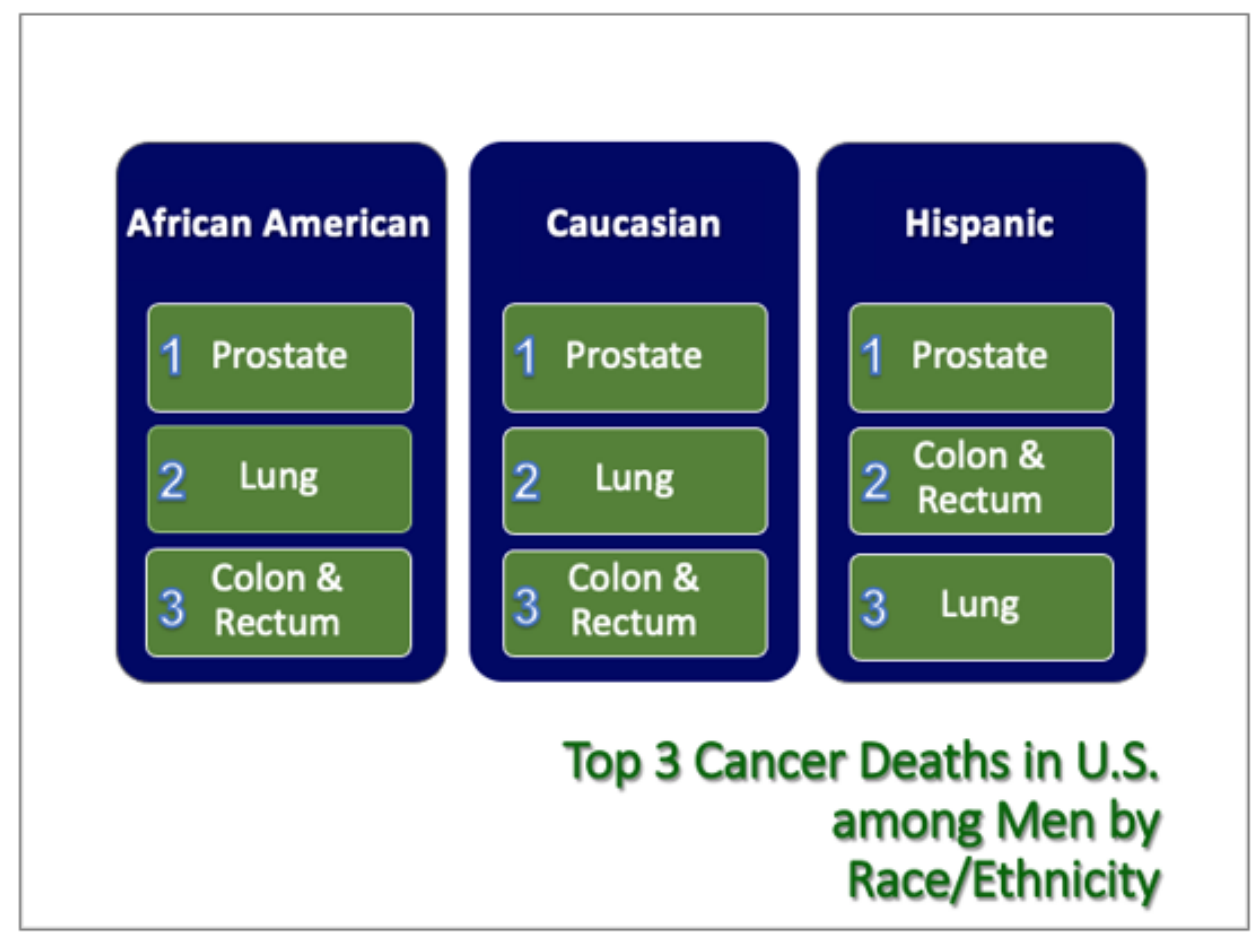

Figure 2

Sample content on clinical trials 